\title{
MRI characteristics of sporadic CJD with valine homozygosity at codon 129 of the prion protein gene and PrPSc type 2 in Japan
}

\author{
R Fukushima, Y Shiga, M Nakamura, J Fujimori, T Kitamoto, Y Yoshida
}

J Neurol Neurosurg Psychiatry 2004;75:485-487. doi: 10.1136/jnnp.2002.00801 1

Two Japanese sporadic Creutzfeld-Jakob disease (sCJD) patients with valine homozygosity at codon 129 of the prion protein gene and protease-resistant prion protein $\left(\mathrm{PrP}^{\mathrm{Sc}}\right)$ type 2 (VV2) are described. In contrast with Western countries, this type of sCJD is very rare in Japan. In 123 sCJD cases, only two were recognised as VV2 by the Japanese CJD surveillance committee. The clinical symptoms and pathological findings of the patients were similar to those of European and US patients. The noteworthy finding of diffusion weighted MRI (DWI) was that an abnormal high intensity covered a wide range of the thalamus including the dorsomedial nucleus, the pulvinar, and the ventral anterior, lateral, and posterolateral nuclei. This thalamic pattern has not been recognised in SCJD with methionine homozygosity and $\mathrm{PrP}^{\mathrm{Sc}}$ type $1(\mathrm{MMI})$ or methionine/valine heterozygosity and $\mathrm{PrPSc}$ type 1 (MV1) which comprises the vast majority of sCJD. This finding may be characteristic to $\mathrm{V} 2$ and may distinguish it from MM1, MVI, and variant CJD. DWI can provide a very important clue for the antemortem diagnosis of VV2 subjects.

S ince the discovery of the relationship between bovine spongiform encephalopathy and variant CreutzfeldtJakob disease (vCJD) in the United Kingdom, ${ }^{1}$ nationwide surveillance for CJD including vCJD has begun in many countries. It has been found that the major differential diagnosis of vCJD is sporadic CJD (sCJD). ${ }^{2}$ The classical triad of progressive dementia, generalised myoclonus, and periodic sharp and slow wave complexes (PSWCs) in electroencephalogram (EEG) are important clues for sCJD. Periodic sharp and slow wave complexes in EEG are a useful marker for distinguishing SCJD from vCJD because vCJD never shows PSWCs. ${ }^{2}$ However, both the differentiation of SCJD without PSWCs from VCJD and the diagnosis of such an uncommon SCJD are difficult.

In 1999, Parchi, et al proposed a classification of SCJD based on a polymorphism at codon 129 of the prion protein gene (PRNP) and the types of protease-resistant prion protein $\left(\mathrm{PrP}^{\mathrm{Sc}}\right){ }^{3} \quad$ Sporadic CJD with type $2 \mathrm{PrP}^{\mathrm{Sc}}$ and valine homozygosity at codon 129 of PRNP (VV2) is a representative SCJD without PSWCs and the second most common type of SCJD in Western countries. ${ }^{3}$ On the other hand, reflecting the fact that valine homozygosity at codon 129 (VV) in the normal Japanese population is very rare, ${ }^{4}$ only one Japanese SCJD with VV has been reported. ${ }^{5}$ We report two cases of Japanese SCJD with VV2 and their characteristic diffusion weighted MRI (DWI) findings which are useful for distinguishing SCJD with VV2 from SCJD with methionine homozygosity (MM) and type $1 \operatorname{PrP}^{\mathrm{Sc}}(\mathrm{MMl})$, methionine/ valine heterozygosity (MV) and type $1 \operatorname{PrP}^{\mathrm{Sc}}(\mathrm{MVl})$, and vCJD.

\section{CASE REPORT}

Patient 1 was a 75 year old woman without a family history of prion disease or personal history of neurological disease. She had never received cadaveric growth hormone, dura mater, or cornea. She noticed unsteadiness of gait in February 1999. She visited our outpatient clinic in March 1999 because of this progressive unsteadiness. Neurological examination showed only cerebellar ataxia. The cerebellar ataxia progressed and mild cognitive dysfunctions appeared. In May 1999, she was unable to walk because of advanced cerebellar ataxia, and dementia rapidly progressed. In July 1999, slight myoclonic jerks appeared and she became akinetic and mute thereafter. She died of pneumonia on 4 January 2000.

Cerebrospinal fluid analysis was normal except for an increased concentration of neuron specific enolase (NSE) (77 ng/ml) and the presence of $14-3-3$ protein (+). Four sequential EEGs showed no PSWCs during her illness. Diffusion weighted MRI showed an abnormal high intensity in the head of the caudate nucleus, putamen, cingurate gyrus, and insular cortex. A wide range of the thalamus was also involved in the lesion (fig 1A). Genetic analysis of codon 129 of PRNP revealed VV and no point mutation was found. Postmortem examination revealed diffuse spongiform changes, gliosis, and neuronal loss in the cortical ribbon except for the calcarine gyrus where these features were limited to the deep layers. These features were also present in the striatum and thalamus, and marked in the cerebellar granular layer with preserved Purkinje cells. PrP deposition occurred in a plaque-like pattern. Western blot analysis revealed type $2 \mathrm{PrP}^{\mathrm{Sc}}$

Patient 2 was a 69 year old man with no family history of prion disease or personal history of neurological disease. He had never received cadaveric growth hormone, dura mater, or cornea. He noticed unsteadiness of gait in June 2001. He could not continue his accounting work from July 2001. Unsteadiness and intellectual deterioration progressed and dysphagia then appeared. He was admitted to our hospital in September 2001. Neurological examination showed mild dementia, frontal release signs, and cerebellar ataxia. He could walk with the aid of a handrail. Soon after admission, he could not remain sitting. Less prominent myoclonic jerks were evoked by extrinsic stimulation from November 2001.

Abbreviations: DWI, diffusion weighted MRI; EEG, electroencephalogram; PRNP, prion protein gene; PrP, prion protein; PSWC, periodic sharp and slow wave complexes; sCJD, sporadic Creutzfeld-Jakob disease; vCJD, variant Creutzfeldt-Jakob disease 

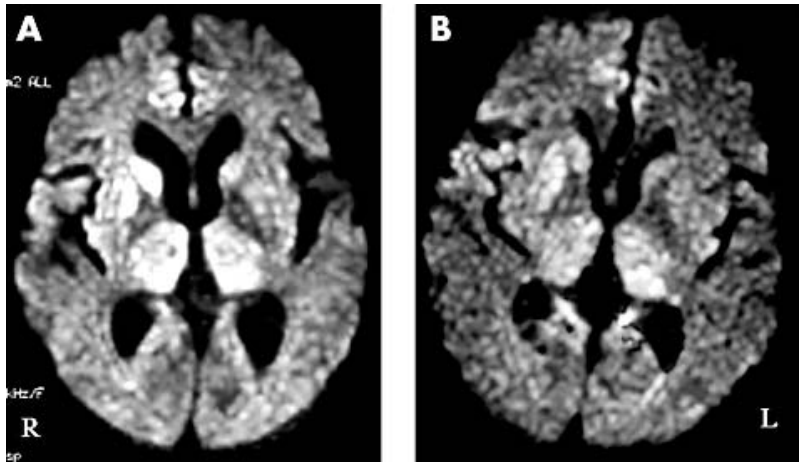

Figure 1 Diffusion weighted MRI (DWI) was performed with single shot spin-echo echo-planar imaging using 1.5-T MR unit (Signa, GE Medical System, Milwaukee, WI, USA). Imaging parameters were as follows: 4999/104/1 (TR/TE/NEX), $5 \mathrm{~mm}$ section thickness with a $1 \mathrm{~mm}$ intersection gap, $128 \times 128$ matrix, $230 \mathrm{~mm}$ field of view, and a diffusion encoding strength (b value) of $1000 \mathrm{~s} / \mathrm{mm}^{2}$. (A) Axial brain DWI of patient 1 showed abnormal high intensity in the head of the caudate nucleus, the putamen, the cingurate gyrus, and the insular cortex. Note that a wide range of the thalamus including the dorsomedial nucleus, the pulvinar, the ventral anterior nucleus, the ventral lateral nucleus, and the posterolateral nucleus were affected by the lesion. (B) Axial brain DWI of patient 2 showed abnormal high intensity in the head of the caudate nucleus, the putamen, and the cortical ribbon similar to patient 1 . Note that a wide range of the thalamus including the dorsomedial nucleus, the pulvinar, the ventral anterior nucleus, the ventral lateral nucleus, and the ventral posterolateral nucleus were affected by the lesion.

He became akinetic and mute in December 2001. He died of pneumonia on 24 January 2002.

Cerebrospinal fluid analysis was normal except for an increased concentration of NSE $(110 \mathrm{ng} / \mathrm{ml})$ and the presence of 14-3-3 protein (++). Three sequential EEGs showed no PSWCs during his illness. Diffusion weighted MRI showed an abnormal high intensity in the head of the caudate nucleus, putamen, and cortical ribbon (prominent in the cingurate gyrus, and insular cortex. Like patient 1 , a wide range of the thalamus was also affected by the lesion (fig 1B). Genetic analysis of codon 129 of PRNP revealed VV and no point mutation was found. In the postmortem examination, spongiform changes, gliosis, and neuronal loss in the cortical ribbon were limited to deep layers and the laminar cortical structure was preserved except for the frontal lobe. These features were also present in the striatum, thalamus, and brainstem nuclei, and marked in the cerebellar granular layer with preserved Purkinje cells. PrP staining mainly showed plaque-like structures. No amyloid plaques were visible. Western blot analysis revealed type $2 \operatorname{PrP}^{\mathrm{Sc}}$.

\section{DISCUSSION}

Our two patients exhibited the uncommon variant of SCJD with VV2. They started with cerebellar ataxia followed by dementia and became akinetic and mute five and six months respectively after the onset. Compared with the mean duration of 3.5 months from onset to becoming akinetic and mute of 116 Japanese typical sCJD with MM (calculated by the authors based on the data reported to Japanese CJD surveillance committee), the five and six months of our patients were relatively longer, but the difference was not statistically significant. Myoclonic jerk was less prominent, and no PSWCs were detected during their illness. These clinical characteristics coincided with those of SCJD with VV2 in Western countries, ${ }^{3}$ the previous ataxic variant. ${ }^{6}$ The pathological features of our patients also coincided with those of Western SCJD with VV2. ${ }^{3}$ According to a report by Parchi et al, 16\% of their sCJD subjects had VV2 and they represented the second most common subtype. However, in Japan, sCJD with VV2 is extremely rare and only one sCJD with VV has been reported.5 Of the 123 sCJD subjects with the genotype at codon 129 reported to the Japanese CJD surveillance committee, 116 subjects had MM, five had MV, and two (the present patients) had VV. These differences relate to the difference of the genotype at codon 129 in the normal population between Japan and Western countries; $92 \%$ for $\mathrm{MM}, 8 \%$ for $\mathrm{MV}$, and $0 \%$ for $\mathrm{VV}$ in Japanese $^{4} v 37 \%$ for MM, 51\% for MV, and $12 \%$ for VV in Westerners. $^{3}$

Another characteristic of our patients was the DWI finding. Diffusion weighted MRI is sufficiently sensitive to show the lesions of $\mathrm{CJD}^{78}$ and helped us diagnose our patients. Usually, hyper-intense lesions are shown in the cortical ribbon and the basal ganglia. "Pulvinar sign" and "Hockey stick sign" are famous MRI findings demonstrating thalamic lesions in $\mathrm{vCJD}^{10}$ and are included in the diagnostic criteria of vCJD. ${ }^{2}$ In our patients, however, DWI showed hyperintense lesions in the cortical ribbon, basal ganglia, and a wide range of the thalamus. These thalamic lesions involved the dorsomedial nucleus, the pulvinar, the ventral anterior nucleus, the ventral lateral nucleus, and the ventral posterolateral nucleus. This thalamic pattern is apparently different from that of VCJD. Various lesions in CJD are depicted on MRI; however, the thalamic lesion is rare. Only $7.4 \%$ of CJD patients demonstrated thalamic lesion and the changes were only mild. ${ }^{11}$ We reviewed the DWI findings of our 24 CJD patients comprising 17 sporadic (including one MM2thalamic based on Parchi's classification ${ }^{3}$ and one MV as uncommon variants), six familial (one substitution of arginine for methionine at codon 232, one substitution of lysine for glutamine at codon 200, and four substitutions of isoleucine for valine at codon 180), and one iatrogenic CJD (a recipient of cadaveric dura mater) in addition to the present two patients. We found basal ganglia lesions in three, cerebral cortical lesions in six, and both lesions in 15 patients, but could not find thalamic lesions. Including the present two patients, two of $26(7.7 \%)$ CJD patients in our series had thalamic lesions on DWI. Schröter et al, ${ }^{11}$ Finkenstaedt et al, ${ }^{12}$ and Samman et $a l^{13}$ described thalamic lesions but they were restricted to the dorsomedial nucleus and pulvinar. Bahn et $a l^{14}$ and Collie et $a l^{15}$ also described thalamic lesions in the dorsomedial nucleus and the pulvinar, respectively. The thalamic lesions that we demonstrated in our two patients involved a wider range of the thalamus than those described in previous reports. ${ }^{11-15}$ Thalamic lesions that involved almost the entire thalamus were in both our patients and may be a characteristic of SCJD with VV2 rather than merely a coincidence, because such thalamic lesions have not been described before and SCJD with VV2 is extremely rare in Japanese.

Diffusion weighted MRI findings are not included among the major diagnostic criteria, ${ }^{16}$ although DWI is more sensitive than EEG and can detect lesions at an early stage. ${ }^{17}$ Even in cases of SCJD without the classical triad, we can make a correct diagnosis more easily if we know the specific imaging finding for a specific subtype. In the case of SCJD with VV2, which does not show prominent myoclonic jerks or PSWCs in the EEG, the characteristic thalamic lesions demonstrated in DWI may lead to an exact diagnosis. These characteristic thalamic lesions can also be an important clue to distinguish sCJD with VV2 from VCJD, because the major differential diagnosis of VCJD is SCJD without PSWCs.

\section{ACKNOWLEDGEMENTS}

We thank Mr Brent Bell for reading the manuscript. 


\section{Authors' affiliations}

R Fukushima, M Nakamura, The Second Department of Internal Medicine, Hiraka General Hospital, Yokote, Japan

Y Shiga, J Fujimori, Departments of Neurology, Tohoku University School of Medicine, Sendai, Japan

T Kitamoto, Departments of Neurological Science, Tohoku University School of Medicine, Sendai, Japan

Y Yoshida, Department of Pathology, Research Institute for Brain and Blood Vessels-Akita, Japan

Y Shiga, T Kitamoto are on the Japanese CJD Surveillance Committee

Correspondence to: Dr R Fukushima, The Second Department of Internal Medicine, Hiraka General Hospital, 1-30 Ekimae-cho, Yokote 013-

8610, Japan; fukutami@rnac.ne.jp

Received 30 November 2002

In revised form 30 June 2003

Accepted 9 July 2003

\section{REFERENCES}

1 Will RG Ironside JW, Zeidler $M$ et al. A new variant of Creutzfeldt-Jakob disease in the UK. Lancet 1996;347:921-5.

2 Will RG, Zeidler M, Stewart GE, et al. Diagnosis of new variant CreutzfeldtJakob disease. Ann Neurol 2000;47:575-82.

3 Parchi P, Giese A, Capellari S, et al. Classification of sporadic CreutzfeldtJakob disease based on molecular and phenotypic analysis of 300 subjects. Ann Neurol 1999;46:224-33.

4 Doh-ura K, Kitamoto T, Sakaki Y, et al. CJD discrepancy. Nature 1991;353:801-2
5 Miyazono M, Kitamoto T, Doh-ura K, et al. Creutzfeldt-Jakob disease with codon 129 polymorphism (Valine): a comparative study of patients with codon 102 point mutation or without mutations. Acta Neuropathol 1992;84:349-54.

6 Brownell B, Oppenheimer DR. An ataxic form of subacute presenile polioencephalopathy (Creutzfeldt-Jakob disease). I Neurol Neurosurg Psychiatry 1965;28:350-61.

7 Damaerel $\mathrm{P}$, Heiner L, Robberecht W, et al. Diffusion-weighted MRI in sporadic Creutzfeldt-Jakob disease. Neurology 1999;52:205-8.

8 Mittal S, Farmer P, Kalina P, et al. Correlation of diffusion-weighted magnetic resonance imaging with neuropathology in Creutzfeldt-Jakob disease. Arch Neurol 2002;59:128-34

9 Zeidler M, Sellar RJ, Collie DA. The pulvinar sign on magnetic resonance imaging in variant Creutzfeldt-Jakob disease. Lancet 2000;355:1412-18.

10 Kretzschmar HA, Ironside JW, DeArmond SJ, et al. Diagnostic criteria for sporadic Creutzfeldt-Jakob disease. Arch Neurol 1996;53:913-20.

11 Schröter A, Zerr I, Henkel K, et al. Magnetic resonance imaging in the Creutzfeldt-Jakob disease. Arch Neurol 2000;57:1751-7.

12 Finkenstaedt M, Szudra A, Zerr I, et al. MR imaging of Creutzfeldt-Jakob disease. Radiology 1996:199:793-8.

13 Samman I, Schulz-Schaeffer WJ, Wöhrle JC, et al. Clinical range and MRI in Creutzfeldt-Jakob disease with heterozygosity at codon 129 and prion protein type2. J Neurol Neurosurg Psychiatry 1999;67:678-81.

14 Bahn MM, Parchi P. Abnormal diffusion-weighted magnetic resonance imaging in Creutzfeldt-Jakob disease. Arch Neurol 1999;56:577-83.

15 Collie DA, Sellar RJ, Zeidler M, et al. MRI of Creutzfeldt-Jakob disease: Imaging features and recommended MRI protocol. Clin Radiology 2001:56:726-39.

16 Report of a WHO consultation. Global surveillance, diagnosis and therapy of human transmissible spongiform encephalopathies. WHO/EMC/ZDI/98.9. World Health Organization: Geneva, 1998.

17 Shiga Y, Asano M, Sato S, et al. Diagnostic importance of diffusion-weighted magnetic resonance imaging for Creutzfeldt-Jakob disease (abstract). Ann Neurol 2002;52(Suppl 1):S53. 